\title{
INDOOR BUILDING SURVEY ASSESSMENT
}

\author{
T. Lovas ${ }^{1 *}$, K. Hadzijanisz ${ }^{2}$, V. Papp ${ }^{2}$, A. J. Somogyi ${ }^{1}$ \\ ${ }^{1}$ Department of Photogrammetry and Geoinformatics, Budapest University of Technology and Economics, Muegyetem rkp. 3, 1111 \\ Budapest, Hungary - (lovas.tamas, somogyi.arpad)@epito.bme.hu \\ ${ }^{2}$ 4iG PLC. Montevideo u. 8. 1037 Budapest, Hungary (konsztantinosz.hadzijanisz, viktor.papp)@4ig.hu
}

\author{
Commission I, WG 7
}

KEY WORDS: indoor mapping, accuracy assessment, mobile mapping systems, cloud-to-cloud evaluation

\begin{abstract}
:
There are multiple emerging technologies, devices and integrated equipment to support indoor mapping. The two main categories are the wearable/portable (e.g. hand-held or backpack devices) and the trolley based devices. The most widely used sensors of the integrated systems are the laser scanners (usually profile scanners), camera(s) and the IMU unit. Compared to outdoor mobile mapping systems the main difference is the lack of GNSS signals; localization is usually supported by SLAM (Simultaneous Localization and Mapping) technology, using Kalman-filtering. Current paper discusses the assessment of the potential of trolleybased indoor mobile mapping systems (MMS) by surveying a building part by multiple technologies. Besides conventional land surveying measurements, terrestrial lasers scanning and a backpack-based mobile survey have been carried out. The analysis included cloud-to-cloud comparison as well as CAD-based evaluation focusing on the geometric accuracy of the point clouds. The paper also presents the surveying workflow; on its resource-needs and potential application fields. The paper discusses the data acquisition technologies and procedures and the different quality assessment methods and results. Since an experimental survey was conducted with a backpack-based unit in the same study area, the paper gives a brief overview on how the two different mobile mapping technologies can be applied indoor, and presents the main differences, advantages and drawbacks.
\end{abstract}

\section{INTRODUCTION}

Indoor mapping can be required by multiple reasons; in many cases design plans, floor plans are not available or the existing ones do not ensure sufficient accuracy, quality or simply not updated. The indoor survey is often complicated and has to be executed rapidly, adapted to the circumstances. Operation of a factory or a hospital cannot be simply suspended to ensure clear line-of-sight to the mapping systems; moving machines and people are disturbing the survey, obstructing the measurements. Therefore the duration of field surveys has to be decreased; this is a key requirement in the development of indoor mapping systems.

The frequently emphasized advantages of these MMSs are that they're fast, reliable and accurate. Overall accuracy is depending on many factors; in 2017 Lehtola et al compared eight mobile laser scanners (MLS) and reviewed their advantages and disadvantages based on operating the instruments and their end product. The compared scanners were Matterport, NavVis (M3), Zebedee, Stencil and Leica Pegasus Backpack. They also reviewed and compared some prototype scanners such us: Alto VILMA, FGI Slammer and the Würzburg backpack. The comparison is based on a test area, which was previously scanned by a terrestrial laser scanner (TLS). There were three different types of test areas: a car park, a hallway and a hall (Lehtola et al, 2017).

For outdoor use, the backpack solution is better, as stated in (Velas et al, 2019). They studied five backpack solutions: LiBackpack (2019), Leica Pegasus Backpack (2015), Viametris bMS3D, Robin (2016), Akhka (2015). The latest versions of trolley-based systems, such as the NavVis M6, are now capable of measuring on slopes (e.g. on ramps) but are still struggling when it comes to stairs. The backpacks, on the other hand, can easily be used to capture stairways and on rough terrains. The main drawback of the backpack system is that it relies on GNSS and when the signal of the satellites is poor or not available, the quality and accuracy drops drastically. This is not a problem for the trollies because it can capture control points or, as they call, anchor points, which can be measured by total stations (Velas et al, 2019).

Filippo et al (2018) reviewed the capability of wearable mobile laser scanners (WMLS) in a complex historical site. Since these systems are for both indoor and outdoor scanning, simultaneous localization and mapping (SLAM) technique is used; when indoors the GNSS signal drops down, mapping still can be continued. In the research, they used the ZEB-REVO backpack WMLS by GeoSLAM that consists of a 2D time-of-flight (TOF) laser scanner (Hokuyo UTM-30LX-F), which is mounted to a rotating engine which has an IMU as well. In the research, they validated the accuracy of the ZEB-REVO with a Faro Focus 3D TLS by measuring the same places and compared the measurements. The whole survey of $3000 \mathrm{~m}^{2}$ with the backpack took 65 minutes. The accuracy of the WLMS was calculated about $3 \mathrm{~cm}$, which is basically the accuracy of the SLAM (Filippo et al, 2018).

Fassi et al (2019) presented a case study of surveying the area of Fort of Pietole in Borgo Virgilio with the Leica Pegasus Backpack. The site has rough, complex terrain, which made it impossible to use trolley based MMS. The aim of the survey was to extract Digital Terrain Model (DTM) of the area (Fassi et al, 2019).

Nocerino et al (2017) investigated two MMS: the ZEB-REVO and the Leica Pegasus Backpack. They measured two typical

\footnotetext{
* Corresponding author
} 
user-case test areas: an indoor two-floor building and an open city square. They compared the two MMS by the surveying experience and the point cloud features and characteristics. The test areas was also surveyed with a Leica HDS7000 TLS and the point clouds have been compared. Their results were within the expectations and the given specifications by the vendors (Nocerino et al, 2017).

Maboudi et al (2017) compared two mobile laser scanner (MLS) with a terrestrial laser scanner (TLS) in order to assess the quality of the devices. The test took place in a controlled environment and they investigated the point cloud's cloud-tocloud distance and the result was within their expectations, the random error was approx. $10 \mathrm{~mm}$. Maboudi et al (2018) also investigated the importance of loop-closure in case of SLAM based instruments. Wen et al in (2014) already published about the importance of loop-closure in case of SLAM and they also compared some of the earliest indoor mobile mapping system (IMMS) models (Wen et al, 2014).

Tucci et al (2018) presented an analytical method to assess the IMMS point clouds. In their research the geometric deformation was computed with Multiscale Model to Model Cloud Comparison (M3C2) algorithm and they also performed cross section and cloud to mesh $(\mathrm{C} 2 \mathrm{M})$ analysis.

Indoor positioning and navigation was always a challenge because the devices lack the GNSS signal to calculate positions accurately. Pathak et al (2014) used wifi signals for localization, but it turned out a dense network of access points would have been needed to triangulate the device.

Another method is proposed by $\mathrm{Wu}$ et al (2018). If a highprecision, photorealistic map is available (e.g. from MMS survey), then the position of the device can be calculated by taking a photo (of for example the corridor) and the software finds the location. Wu et al (2018) studied this method and found that $70 \%$ of the images achieved a location error smaller than $0.9 \mathrm{~m}$ in a $10 \mathrm{~m} \times 15.8 \mathrm{~m}$ room.

The NavVis also developed a similar method to make the indoor navigation possible with their IndoorViewer system. The IndoorViewer is a platform where those who have access can view the panorama images and point clouds of the measured building; they can also make measurements on the point cloud (NavVis, 2019). In a complex building, it is difficult to navigate with 2D maps and carry out positioning and localization, stated in Shuangfeng et al (2019). They investigated the possibility of making a 3D indoor navigation app, using the NavVis' IndoorViewer system. The test area was the library of Beijing University of Civil Engineering and Architecture. They successfully created an application of book localization and retrieval system (Shuangfeng et al, 2019).

The main advantage of the MMS, regardless it is handheld, trolley or backpack, is that it is very fast and easy to use, and no or minimal special knowledge is required to operate these instruments. This is why it has great potential in scan-to-BIM applications.

Campi et al (2018) presented the method of using MMS for architectural use; their study gives an insight of the requirements of data acquisition and they also presented a comparison between static and dynamic scanning (Campi et al, 2018).

NavVis enabled to use its product with the Autodesk Revit (a BIM based software), by installing an add-on to Revit; now it is possible to view the panorama images in the model itself (NavVis, 2020).

Bassier et al (2015) compared TLS and MMS measurements. They used two TLS devices: Leica ScanStation P20 and a Faro Focus3D X330; and three MMS: ZEB1 handheld MMS, Viametris and NavVis M3 trolley based MMS. Their result of the comparison is that while the TLS is slower than the MMS, but its high accuracy is still keeping it as a prominent technique for scan-to-BIM use. Shafiq et al (2018) discussed that the upto-date information of a building has an important role in the facility management (FM); the efficient integration of BIM and FM, aided by MMS results in cost- and energy-saving (Shafiq et al, 2018).

\section{INDOOR MEASUREMENTS}

The primary goal of the current research is to assess the overall potential of a trolley-based indoor mapping system. The potential of technology and its quality assessment is presented based on multiple surveys (1. MMS-trolley + total station, 2 . TLS + total station, 3. MMS-backpack) carried out in the main building of the Budapest University of Technology and Economics, in 2019. The key point of our approach that we investigate multiple technologies in the same area where ground truth is available and that we evaluate the advantages and shortcomings of the trolley and backpack-based solutions this way.

As ground truth, total station (Trimble M3) and TLS (recently considered as a mature technology) were used: selected areas were scanned by a Faro Focus 120S TLS. The tie point coordinates were captured by total station, using the available local geodetic network in the University building.

The NavVis M6 has four laser scanners. The one on the top (Velodyne scanner with $80 \mathrm{~m}$ measurement range) is responsible for the SLAM alignment and the others collecting the data. Point cloud coloring is supported by 6 RGB cameras with fish-eye optics. Data acquisition speed is $1.5 \mathrm{~m} / \mathrm{s}$; however, it is recommended to stop when taking the images. The measuring time for a single dataset should not exceed 3540 minutes; the NavVis also notifies through a pop-up window during the measurement if this time is over. Matching point clouds is solved by anchor points that can be either dedicated marked tie points or existing (natural) tie points. In the postprocessing phase, the NavVis performs some filtering, which results that the point cloud is clean and smooth.

Prior to the NavVis measurement, a survey plan was created that split the entire area to 9 survey blocks. The survey plan also supported to define the locations of the tie points that are used to align/register the datasets (77 datasets in total). Before the actual survey, the area was cleared by removing objects considering privacy issues or avoiding obstruction. The whole floor's $\left(5000 \mathrm{~m}^{2}\right)$ surveying took around 3 hours and 52 minutes and consisted of 170 million points. The postprocessing time is basically depending on the surveying time. A 30 minute dataset's post-processing takes around 3-5 hours with the sufficient hardware requirements. In comparison, an over 40 minute survey's processing time can be more than 10 hours with the same computational capacity. Obviously, the processing can be shared between computers to reduce the computation time.

During the survey, the NavVis trolley was traveling the planned route and capturing all the dedicated tie points. The data process was carried out in two steps; postprocess (deriving trajectory, raw point cloud, panoramic images, colored point cloud) and webprocess (preparing the datasets for the NavVis IndoorViewer, files for web-based publishing).

\section{ASSESSING INDOOR SURVEYING TECHNOLOGIES TO THE GROUND TRUTH}

Deviations between the checkerboard (used as tie points, marked by F1001 to F1009) centerpoint coordinates derived 
from total station measurements and that of the NavVis survey have been determined and analyzed.

Figure 1 shows the deviation values. It seems that higher values can be observed in $\mathrm{Z}$ direction (height) and in $\mathrm{Y}$ direction, that is the traveling direction of the trolley. On the other hand, $X$ values (towards the course of the normal of the boards) are minimal.

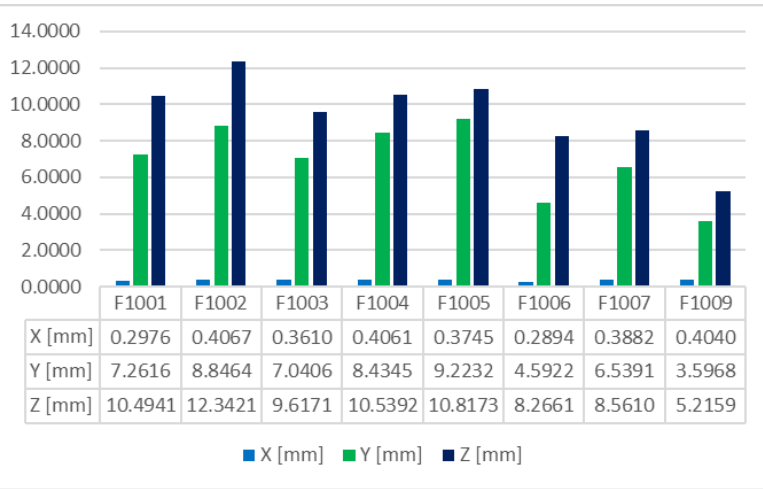

Figure 1 Deviations on checkerboards

To evaluate the potential of the technologies for architectural purposes, deviation maps have been created for both the NavVis (Figure 3) and Leica (Figure 4) point clouds; TLS point clouds were used as ground truth. Matching the point clouds was supported by tie points in case of NavVis, while cloud-tocloud fitting was used in case of the Leica datasets, since dedicated tie points were no longer available during the latter survey. In both cases most deviations are below $1.5 \mathrm{~cm}$. While Leica deviations seem homogeneous, the scan profiles are clearly visible in case of NavVis (Figure 2). Point density and accuracy enables deriving architectural products (views, sections, floorplans) with the scale of 1:50 (or smaller) using appropriate vectorizing methods.

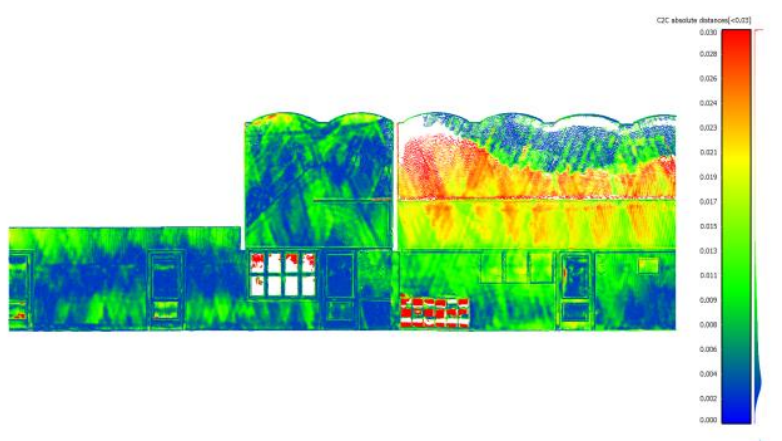

Figure 2 Deviation map for NavVis point cloud

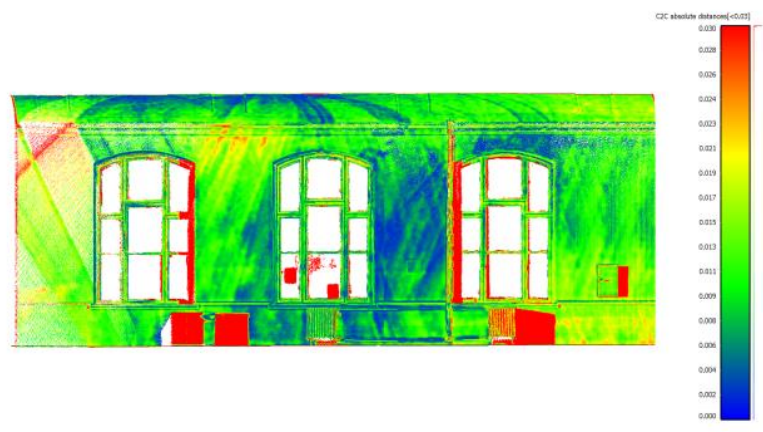

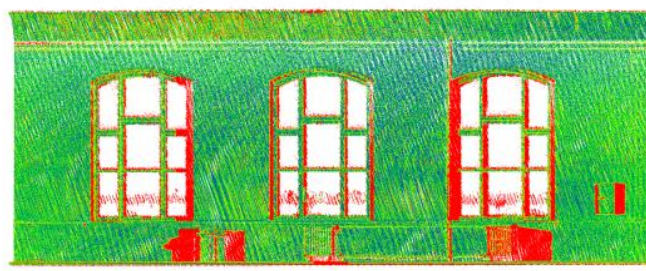

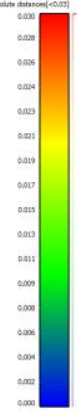

Figure 4 Deviation map for Leica point cloud - details

In case of complex geometry is to be mapped (e.g. in a factory hall with machines), capturing fine details can be a crucial requirement. Therefore we surveyed a sculpture with both TLS and MMS in order to evaluate the potential of MMS for such purposes.

Figure 5 clearly shows that the backpack-based solution provides a sparse point cloud (right sub-figure) compared to that of the Navvis (middle) and TLS (left). Deviations remain under $1.5 \mathrm{~cm}$, NavVis has lower values.

Regarding modeling potential, both technologies are capable of supporting low LOD (level of detail/development) modeling; however, currently none of them enables creating high density models with fine details.
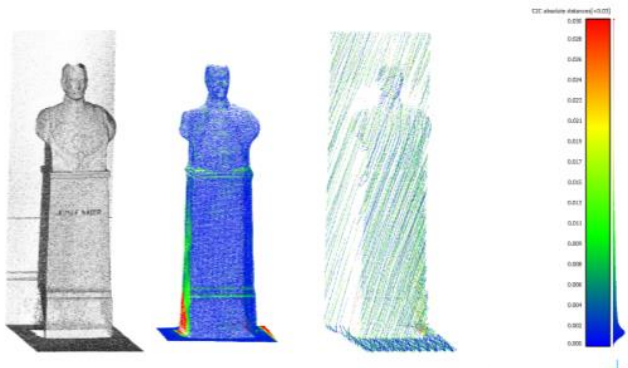

Figure 5 Sculpture point clouds - TLS (left), NavVis (middle),

$$
\text { Leica (right) }
$$

In case of NavVis we checked the derived corridor crosssections (taken perpendicular to MMS' traveling direction) and compared to the reference sections (from TLS). While deviation values are mostly below $1.5 \mathrm{~cm}$, representing complex geometry is problematic in the NavVis point cloud, some architectural details tend to be smoothened (Figure 6).

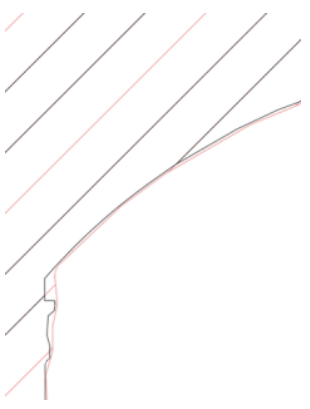

Figure 6 Capturing architectural details with TLS (black) and MMS-NavVis (red)

Figure 3 Deviation map for NavVis point cloud - details 


\section{COMPARING INDOOR SURVEYING TECHNOLOGIES}

Hereby we review the point clouds produced by the two (NavVis and Leica Pegasus Backpack) MMS. First we present the point clouds separately, showing their characteristics and properties, then we analyze them by calculating their cloud-tocloud distance and measuring distances in their cross-sections. For the analyses we used CloudCompare, an open source software for point cloud manipulation and analyses.

First we analyzed the Pegasus Backpack's point cloud (Figure 7). This instrument has two laser scanners, one for the SLAM alignment (mounted on the top of the backpack) and the other (mounted on the back of the backpack) is for the data recognition. Since the second scanner facing the ground not precisely perpendicularly, the footprint of the scanner has circular shape. Since only a single scanner is collecting data it is less dense than that of the NavVis. It is important to note that both surveys were carried out by their most typical settings (e.g. walking/trolley speed), the goal was not to reach the potentially best point cloud but to represent the typical application circumstances. This is the main reason why the Backpack's point cloud was sparser compared to that of the NavVis is (Figure 8).

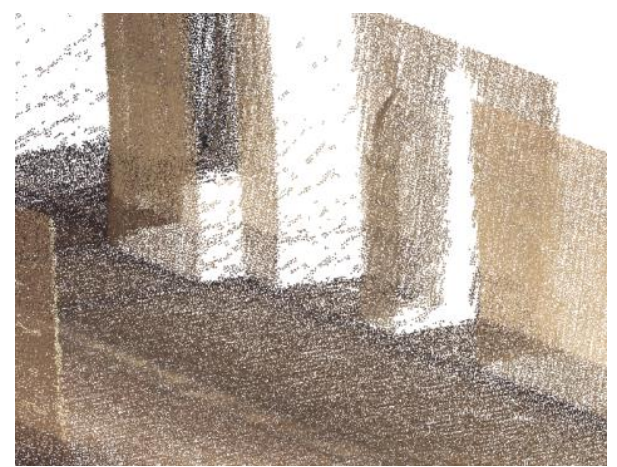

Figure 7 Leica Pegasus backpack point cloud

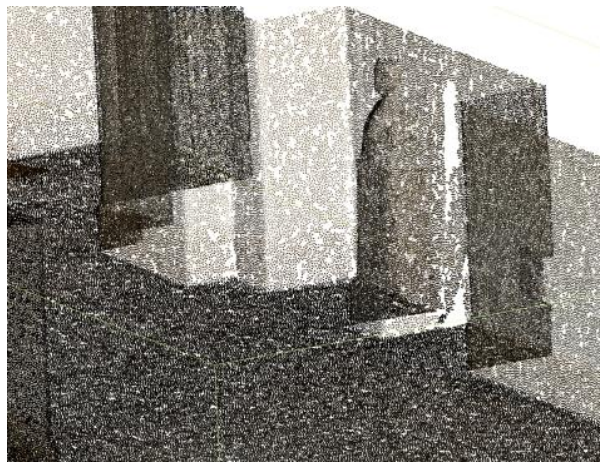

Figure $8 \mathrm{NavVis} \mathrm{M6} \mathrm{point} \mathrm{cloud}$

The first analysis was comparing the point clouds of the two surveys. First, we imported the point clouds into CloudCompare and clipped the same area of each. There were two different areas analyzed; the first was an open corridor which goes around an open hall. This was selected because each instrument has the highest accuracy here since their scanners, which is responsible for the alignment has a range of $100 \mathrm{~m}$, recorded points from all sides of the space. The second area was a long (around $80 \mathrm{~m}$ ) corridor, which was selected because we believed it is the most challenging area for both MMS regarding accuracy.
In order to save computation time we segmented the point cloud to a smaller area,. For this smaller part of the corridor we resampled the point cloud for $5 \mathrm{~mm}$. After that process the Pegasus point cloud consisted of 437864 points, while the M6 point cloud had 384538 points. Then we executed the cloud-tocloud distance computation, first with $5 \mathrm{~cm}$ maximum threshold, whichindicates the maximal distance the algorithm measures during the process. So in our case every point that is in the red area is at $5 \mathrm{~cm}$ or farther from the other point cloud. Then we repeated the analysis for 3 and $1 \mathrm{~cm} ; 1 \mathrm{~cm}$ threshold doesn't provide reasonable results since most points fell in the red area; the most diverse results were between 5 and $3 \mathrm{~cm}$ thresholds (Figure 9). The results show that the test area's upper part has higher errors than the other parts, however most of the points are in the $1.5 \mathrm{~cm}$ class. This means that both point clouds are mostly on the same level of accuracy.
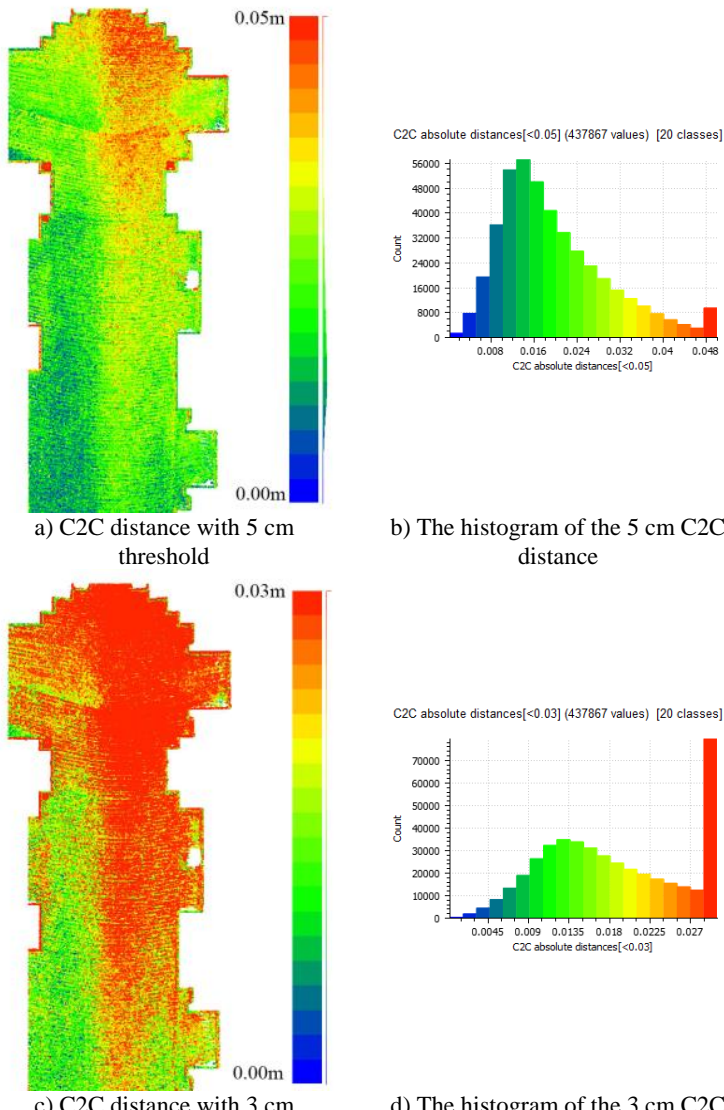

b) The histogram of the $5 \mathrm{~cm} \mathrm{C} 2 \mathrm{C}$ distance

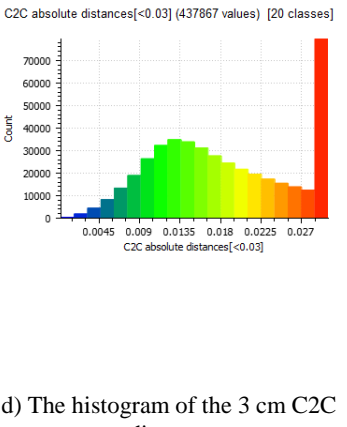

distance

Figure 9 Cloud to cloud (NavVis and Leica) computation top view results in CloudCompare (open corridors)

The main reason of these results is that the Pegasus accuracy in the indoor environment decreases along time because the GNSS signal drops down and it only relies on SLAM alignment and its accuracy is about $5 \mathrm{~cm}$ to $50 \mathrm{~cm}$, based on the Pegasus' documentation (Leica Geosystems, 2017).

We repeated this process for the long corridor to see the differences; the results show that the accuracy drops down, the backpack's point cloud of the corridor bends compared to that of the M6. The NavVis uses anchor points which lowers the bending effect if they are well placed (Figure 10). 

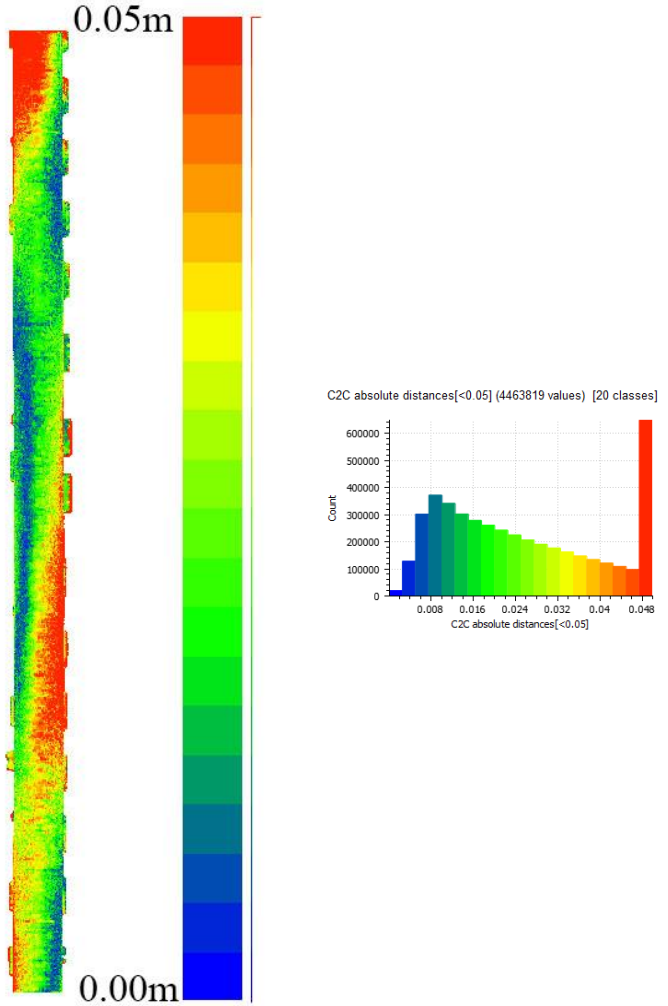

Figure 10 Cloud to cloud (NavVis and Leica) computation top view results in CloudCompare (long corridor)

The results show that the corridor's wall on the point cloud is the critical area because of the bending effect. Also, if we check the inside of the corridor's point cloud it can be seen that the ground points are relatively in good position (in $\mathrm{Z}$ direction) (Figure 11).

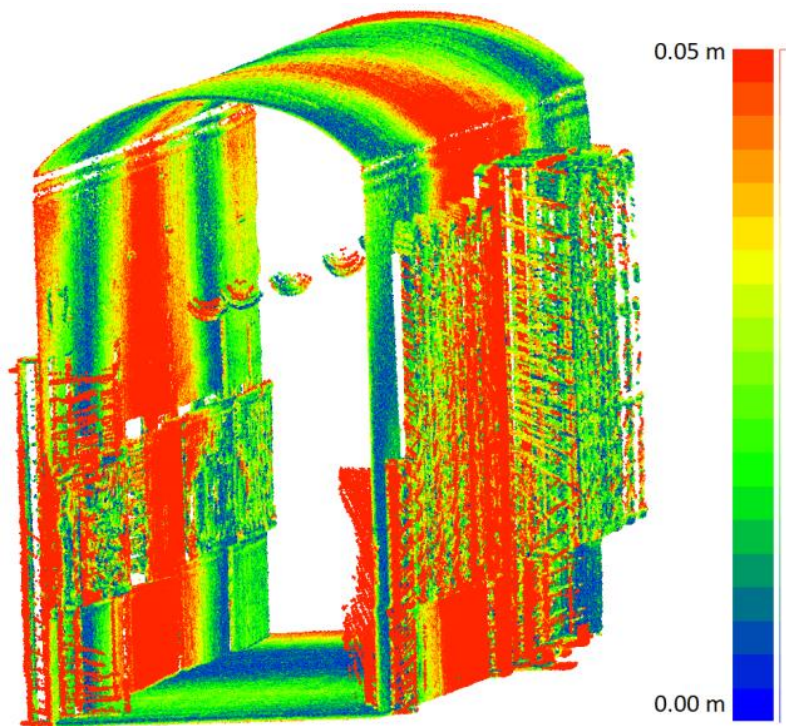

Figure 11 Cloud to cloud (NavVis and Leica) computation (3D) results in CloudCompare (long corridor)

This effect occurs mainly because the lack of constraints and the accuracy of the SLAM alignment drops down. To avoid this problem, the NavVis uses anchor points which can be also measured with total stations to enhance the accuracy of the instrument.
We investigated 3 key cross sections (Figure 12) of the corridor's point clouds to measure the deviations (between sections derived from NavVis and Leica point clouds) manually in CAD environment. Note that the NavVis point cloud is smoother due to the filters it applies during the post processing phase. The key sections were in the two end of the corridor and in the middle.

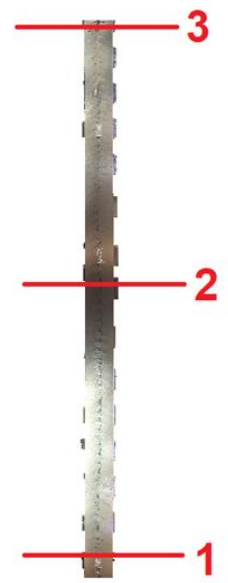

Figure 12 Selected cross sections on the long corridor
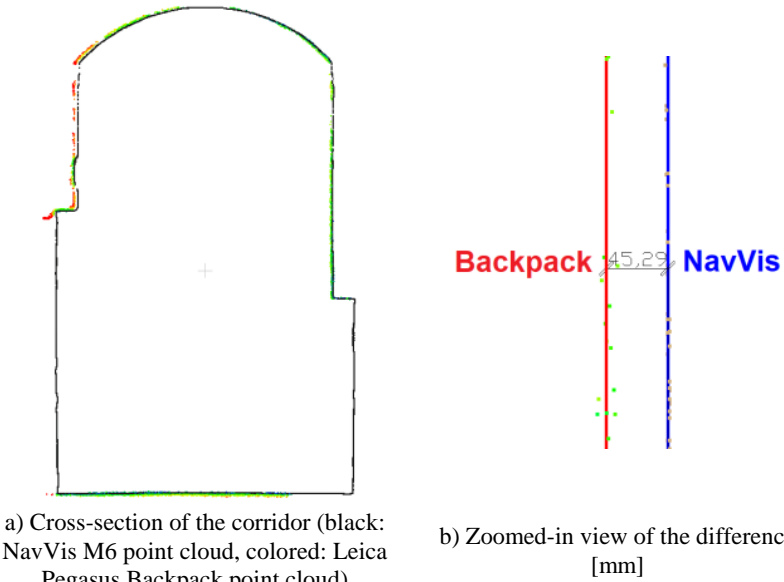

b) Zoomed-in view of the difference $[\mathrm{mm}]$

Figure $131^{\text {st }}$ cross-section of the corridor's point cloud

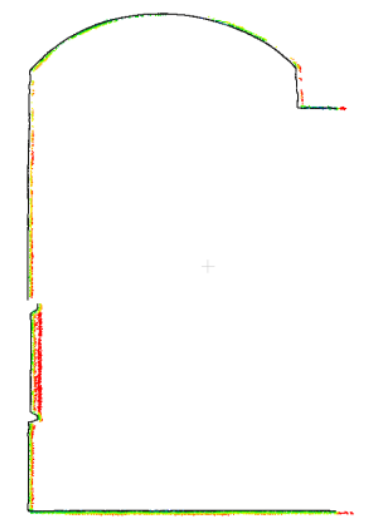

a) Cross-section of the corridor (black: NavVis M6 point cloud, colored: Leica Pegasus Backpack point cloud)

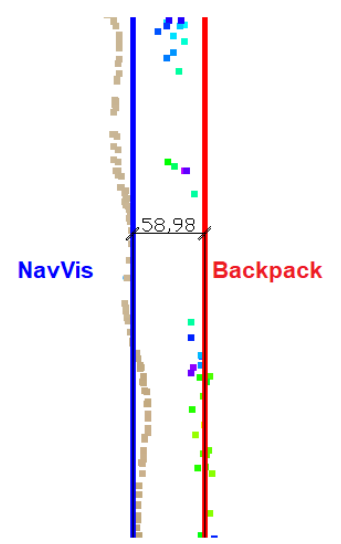

b) Zoomed-in view of the difference [mm]
Figure $142^{\text {nd }}$ cross-section of the corridor's point cloud 


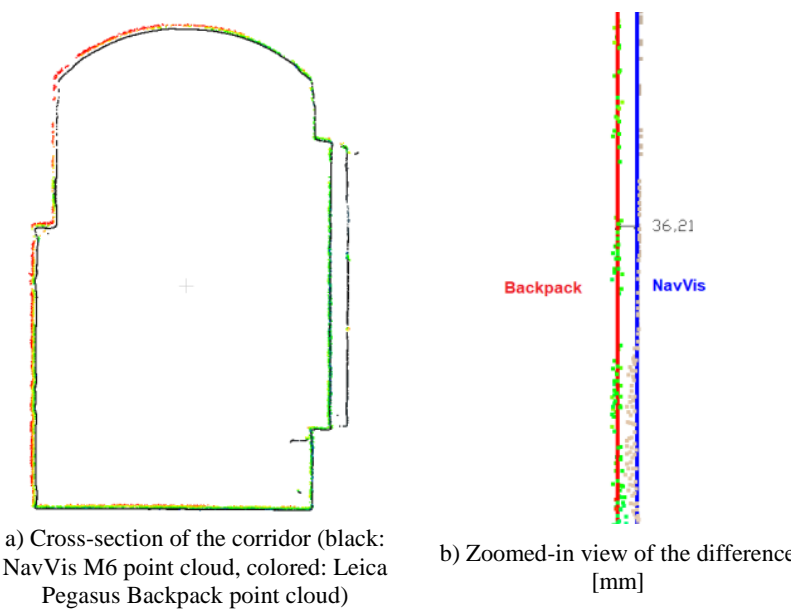

Figure $153^{\text {rd }}$ cross-section of the corridor's point cloud

From the results it is clear that higher errors occur in the $1^{\text {st }}$ (Figure 13) and $3^{\text {rd }}$ (Figure 15) sections, however in the middle section (Figure 14) there are some notable errors as well, but they are in those areas where the scanners struggle to collect data due to non-ideal circumstances. It is also visible that the ceiling and the walls are mostly affected by the bending effect; the ground points are primarily correct, which indicates that no elevation anomaly occurs.

\section{CONCLUSION}

Both investigated MMS technologies are easy to operate by one surveyor and are capable of rapidly provide dense point clouds indoor. From the results it is clear that NavVis M6's advantage over the Leica Pegasus Backpack is that it uses anchor points, which increases its alignment accuracy and lowers the bending effect in case of long corridors. In a more open space, where the SLAM is fairly accurate, both sensors produced datasets with similar accuracy. The Pegasus Backpack's main advantage over the M6 is that it is a backpack; it is more mobile (to store and carry) and easier to survey stairways.

Considering point density and overall geometric accuracy, both systems can effectively support deriving architectural products. According to deviation assessment Pegasus provides more homogeneous pattern with less accuracy (due to the lack of tie points), while circular pattern can be observed in the NavVis dataset. Since the surveys can be executed rapidly both technologies can be used for monitoring purposes. Obviously such systems also support non-engineering applications by providing colored point clouds

Both scanners have ups and downs, it depends mainly on the environment that needs to be surveyed but it can be stated that due to enhanced sensor and algorithm development there are even more solutions to capture indoor geometry for multiple purposes.

\section{ACKNOWLEDGEMENTS}

The Authors are grateful to 4iG PLC. and Leica Geosystems Hungary $\mathrm{Kft}$. for providing the equipment for the test measurements.

\section{REFERENCES}

Shafiq Mohammad \& Sadik Ali Syed BIM for Existing Buildings and its effects on Facility Management. Master's thesis, Göteborg, Sweden, 2018
Nocerino, Erica \& Menna, Fabio \& Remondino, Fabio \& Toschi, Isabella \& Rodríguez-Gonzálvez, Pablo. (2017). Investigation of indoor and outdoor performance of two portable mobile mapping systems. $103320 \mathrm{I}$. doi.org/10.1117/12.2270761.

Wen, Chenglu \& Qin, Ling \& Zhu, Qingyuan \& Wang, Cheng \& Li, Jonathan. (2014). Three-Dimensional Indoor Mobile Mapping With Fusion of Two-Dimensional Laser Scanner and RGB-D Camera Data. Geoscience and Remote Sensing Letters, IEEE. 11. 843-847. doi.org/10.1109/LGRS.2013.2279872.

Fassi, F. and Perfetti, L.: BACKPACK MOBILE MAPPING SOLUTION FOR DTM EXTRACTION OF LARGE INACCESSIBLE SPACES, Int. Arch. Photogramm. Remote Sens. Spatial Inf. Sci., XLII-2/W15, 473-480, doi.org/10.5194/isprs-archives-XLII-2-W15-473-2019, 2019.

Maboudi, Mehdi \& Bánhidi, Dávid \& Gerke, Markus. (2017). Evaluation of indoor mobile mapping systems.

Maboudi, Mehdi \& Bánhidi, Dávid \& Gerke, Markus. (2018). INVESTIGATION OF GEOMETRIC PERFORMANCE OF AN INDOOR MOBILE MAPPING SYSTEM. ISPRS International Archives of the Photogrammetry, Remote Sensing and Spatial Information Sciences. XLII-2. 637-642. doi.org/10.5194/isprs-archives-XLII-2-637-2018.

C. Wen, L. Qin, Q. Zhu, C. Wang and J. Li, "ThreeDimensional Indoor Mobile Mapping With Fusion of TwoDimensional Laser Scanner and RGB-D Camera Data," in IEEE Geoscience and Remote Sensing Letters, vol. 11, no. 4, pp. 843847, April 2014.

Tucci, G.; Visintini, D.; Bonora, V.; Parisi, E.I. Examination of Indoor Mobile Mapping Systems in a Diversified Internal/External Test Field. Appl. Sci. 2018, 8, 401.

Wei, S., Li, B., Guo, Z., Guo, S., and Cheng, L.: A BOOK RETRIEVAL AND LOCATION SYSTEM BASED ON REAL-SCENE 3D, Int. Arch. Photogramm. Remote Sens. Spatial Inf. Sci., XLII-2/W13, 903-908, doi.org/10.5194/isprsarchives-XLII-2-W13-903-2019, 2019.

Campi, M., di Luggo, A., Monaco, S., Siconolfi, M., and Palomba, D.: INDOOR AND OUTDOOR MOBILE MAPPING SYSTEMS FOR ARCHITECTURAL SURVEYS, Int. Arch. Photogramm. Remote Sens. Spatial Inf. Sci., XLII-2, 201-208, doi.org/10.5194/isprs-archives-XLII-2-201-2018, 2018.

Bassier, M., Yousefzadeh, M., \& Genechten, V. B. (2015). Evaluation of data acquisition techniques and workflows for Scan to BIM. Geo Business, At London, (old.: 25). London.

NavVis. (2019). 3D Building in your browser. NavVis official website: https://www.navvis.com/indoorviewer

NavVis. (2020). BIM meets reality. NavVis official website : https://www.navvis.com/revit

Pathak, O., Palaskar, P., Palkar, R., \& Tawari, M. (2014). Wi-Fi Indoor Positioning System Based on RSSI Measurements from Wi-Fi Access Points -A Tri-lateration Approach. International Journal of Scientific \& Engineering Research, 1234-1238. ISSN 2229-5518 
Wu, T., Liu, J., Li, Z., Liu, K., \& Xu, B. (2018). Accurate Smartphone Indoor Visual Positioning Based on a HighPrecision 3D Photorealistic Map. Sensors, 21. doi.org/10.3390/s18061974

Filippo, d. A., Sánchez-Aparicio, J. L., \& Barba, S. (2018). Use of a Wearable Mobile Laser System in Seamless Indoor 3D Mapping of a Complex Historical Site. Remote Sensing, 19. doi.org/10.3390/rs10121897

Lehtola, V. V., Kaartinen, H., Nüchter, A., Kaijaluoto, R., Kukko, A., Litkey, P., . . . Hyyppa, J. (2017). Comparison of the Selected State-Of-The-Art 3D Indoor Scanning and Point Cloud Generation Methods. Remote Sensing, 26. doi.org/10.3390/rs9080796

Velas, M., Spanel, M., Sleziak, T., Habrovec, J., \& Herout, A. (2019). Indoor and Outdoor Backpack Mapping with Calibrated Pair of Velodyne LiDARs. Sensors, 35. doi.org/10.3390/s19183944 